\title{
Error-Prone and Error-Free Translesion DNA Synthesis over Site-Specifically Created DNA Adducts of Aryl Hydrocarbons (3-Nitrobenzanthrone and 4-Aminobiphenyl)
}

\author{
Takashi Yagi ${ }^{1}$, Yoshihiro Fujikawa ${ }^{1,4}$, Tomoko Sawai ${ }^{1}$, Takeji Takamura-Enya ${ }^{2}$, \\ Sayoko Ito-Harashima ${ }^{1}$ and Masanobu Kawanishi ${ }^{1}$ \\ 'Department of Biology, Graduate School of Science, Osaka Prefecture University, Sakai, Osaka, Japan \\ ${ }^{2}$ Department of Applied Chemistry, Kanagawa Institute of Technology, Atsugi, Kanagawa, Japan
}

\begin{abstract}
Aryl hydrocarbons such as 3-nitrobenzanthrone (NBA), 4-aminobiphenyl (ABP), acetylaminofluorene (AAF), benzo(a)pyrene (BaP), and 1-nitropyrene (NP) form bulky DNA adducts when absorbed by mammalian cells. These chemicals are metabolically activated to reactive forms in mammalian cells and preferentially get attached covalently to the $N^{2}$ or C8 positions of guanine or the $N^{6}$ position of adenine. The proportion of $N^{2}$ and $\mathrm{C} 8$ guanine adducts in DNA differs among chemicals. Although these adducts block DNA replication, cells have a mechanism allowing to continue replication by bypassing these adducts: translesion DNA synthesis (TLS). TLS is performed by translesion DNA polymerases-Pol $\eta, \kappa, 1$, and $\zeta$ and Rev1 - in an error-free or error-prone manner. Regarding the NBA adducts, namely, 2-(2'-deoxyguanosin- $N^{2}$-yl)-3-aminobenzanthrone (dG- $\left.N^{2}-\mathrm{ABA}\right)$ and $N$-(2'-deoxyguanosin-8-yl)-3-aminobenzanthrone (dG-C8-ABA), dG- $N^{2}-\mathrm{ABA}$ is produced more often than dG-C8-ABA, whereas dG-C8-ABA blocks DNA replication more strongly than dG- $N^{2}-\mathrm{ABA}$. dG- $\mathrm{N}^{2}$-ABA allows for a less error-prone bypass than dG-C8ABA does. Pol $\eta$ and $\kappa$ are stronger contributors to TLS over dG-C8-ABA, and Pol $\kappa$ bypasses dG-C8$\mathrm{ABA}$ in an error-prone manner. TLS efficiency and error-proneness are affected by the sequences surrounding the adduct, as demonstrated in our previous study on an ABP adduct, $N$ - $(2$ '-deoxyguanosine-8yl)-4-aminobiphenyl (dG-C8-ABP). Elucidation of the general mechanisms determining efficiency, errorproneness, and the polymerases involved in TLS over various adducts is the next step in the research on TLS. These TLS studies will clarify the mechanisms underlying aryl hydrocarbon mutagenesis and carcinogenesis in more detail.
\end{abstract}

Key words: Aryl hydrocarbon, DNA adduct, Translesion DNA synthesis, 3-Nitrobenzanthrone, Mutation

Correspondence to: Takashi Yagi, Department of Biology, Graduate School of Science, Osaka Prefecture University, 1-2 Gakuencho, Naka-ku, Sakai, Osaka 599-8570, Japan

E-mail: yagi-t@riast.osakafu-u.ac.jp

"Present Address: Department of Radiation Biology and Medical Genetics, Graduate School of Medicine, Osaka University, Suita, Osaka, Japan

This is an Open-Access article distributed under the terms of the Creative Commons Attribution Non-Commercial License (http:// creativecommons.org/licenses/by-nc/3.0) which permits unrestricted non-commercial use, distribution, and reproduction in any medium, provided the original work is properly cited.
Abbreviations: AAF, acetylaminofluorene; ABP, 4-aminobiphenyl; $\mathrm{AP}$, apurinic/apyrimidinic; BaP, benzo(a)pyrene; $\mathrm{BER}$, base excision repair; BPDE, ( \pm )-anti-benzo[a]pyrene-7,8-diol-9,10-epoxide; IPTG, isopropyl- $\beta$-D-thiogalactopyranoside; IQ, 2-amino-3-methylimidazo-[4,5-f]quinoline; $N$-Aco-ABA, $N$-acetoxy-3-aminobenzanthrone; dG-C8-ABP, $\quad N$-(2'-deoxyguanosine-8-yl)-4-aminobiphenyl; dG- $N^{2}$ ABA, 2-(2'-deoxyguanosin- $N^{2}$-yl)-3-aminobenzanthrone; dG-C8-ABA, $\mathrm{N}$-(2'-deoxyguanosin-8-yl)-3-aminobenzanthrone; NBA, 3-nitrobenzanthrone; NER, nucleotide excision repair; NP, 1-nitropyrene; TLS, translesion DNA synthesis; X-gal, 5-bromo-4-chloro-3-indolyl$\beta$-D-galactopyranoside; XPA, xeroderma pigmentosum complementation group $A$. 


\section{INTRODUCTION}

Some mutagens have reactive groups such as aldehyde and methyl in their structures, covalently bind to naked or cellular DNA via a nucleophilic reaction, and are called direct mutagens. Other mutagens are metabolically transformed to reactive intermediates in the detoxification process after mutagens are absorbed by cells, get covalently attached to cellular DNA, and are called indirect mutagens. In DNA, mutagens covalently bind to one of several positions mostly in guanine and adenine. Alkylating agents, such as methyl methanesulfonate, $N$-methyl- $N$ '-nitrosoguanidine, and $N$-methyl nitrosourea, preferentially donate methyl groups to the N7 or N3 position of guanine or adenine or the $O^{6}$ position of guanine. Aryl hydrocarbons such as benzo(a)pyrene $(\mathrm{BaP})$ and 1,8-dinitropyrene are metabolically activated, and their metabolites preferentially get attached covalently to the $N^{2}$ or C8 position of guanine. There are numerous kinds of mutagens in the environment; however, their molecular pathways to DNA adduct formation have not yet been clarified in detail.

In this review, the mechanisms underlying the mutations caused by environmental aryl hydrocarbons, particularly 3nitrobenzanthrone (NBA) and 4-aminobiphenyl (ABP), are described. These compounds are biotransformed into reactive forms by phase I and II cellular metabolic enzymes. The formation and repair of their DNA adducts in cells are also described. Although these DNA adducts block DNA replication, cells have a mechanism allowing to continue replication by bypassing these adducts by means of specific DNA polymerases, and this process is called translesion DNA synthesis (TLS). TLS over the adducts derived from NBA and ABP is discussed using data obtained by our original TLS assay.

\section{FORMATION AND REPAIR OF BULKY DNA ADDUCTS}

Most aryl hydrocarbons known as environmental pollutants are indirect mutagens, which are transformed to reactive forms by detoxification enzymes in some cells. The process of biotransformation of $\mathrm{BaP}$, which is abundant in the atmosphere and released during oil combustion, has been elucidated in detail (1). BaP binds to aryl hydrocarbon receptors, and functions as a transcription factor to induce CYP1 family genes in cells. Induced CYP1A1 and CYP1B1 enzymes hydroxylate a benzene ring and eventually transform $\mathrm{B}(\mathrm{a}) \mathrm{P}$ to $( \pm)$-anti-benzo[a]pyrene-7,8-diol-9,10-epoxide (BPDE). BPDE mainly binds covalently to the $N^{2}$ position of guanine (2-4).

NBA is an air pollutant that originates from the diesel exhaust of cars and factories and a strong mutagen like 1,8dinitropyrene in the Ames bacterial mutation assay (5). NBA is primarily processed by cytosolic nitroreductases, followed by activation by $N$-acetyltransferase and sulfotransferase, leading to the formation of a reactive intermediate, $N$-acetoxy-3-aminobenzanthrone ( $N$-Aco-ABA), which forms covalent bonds with guanine and adenine $(6,7)$. These adducts are 2-(2'-deoxyguanosin- $N^{2}$-yl)-3-aminobenzanthrone (dG- $N^{2}$-ABA), $N$-(2'-deoxyguanosin-8-yl)-3-aminobenzanthrone (dG-C8-ABA), and 2-(2'-deoxyadenosin- $\left.N^{6}-\mathrm{yl}\right)-3-$ aminobenzanthrone (dA- $\left.N^{6}-\mathrm{ABA}\right)$, listed in the order of their amounts $(8,9)$ (Fig. 1). NBA causes lung cancer in experimental animals (10).

ABP is mainly generated by cigarette smoking and combustion of fossil fuels and is also a byproduct of the rubber, coal, textile, and printing industries (11). ABP has been identified as a major etiological agent of human bladder cancer and is a potent urinary-bladder carcinogen in experimental animals $(12,13)$. ABP is metabolically activated by cellular $N$-acetyltransferase and forms a major DNA adduct, $N$-(2'-deoxyguanosine-8-yl)-4-aminobiphenyl (dG-C8-ABP) (Fig. 1). The mutation of the $p 53$ tumor suppressor gene in bladder carcinomas of patients who are occupationally exposed to ABP has been examined, and p53 mutation hot spots coincide with the ABP-adducted sites of the $p 53$ gene in cells experimentally exposed to ABP (14).

In the cell, bulky DNA lesions including NBA and ABP adducts are removed by the nucleotide excision repair (NER) pathway, which involves dual incisions of adducted DNA strands, removal of the incised strand, and semicon-

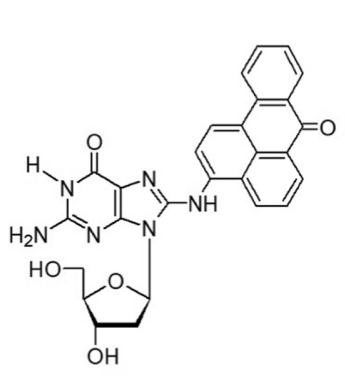

dG-C8-ABA

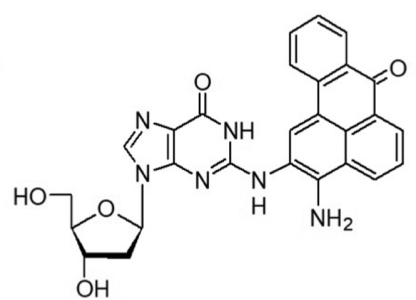

dG- $N^{2}-A B A$

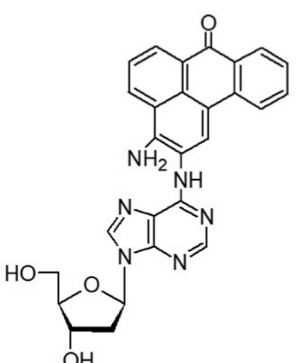

$\mathrm{dA}-N^{6}-\mathrm{ABA}$

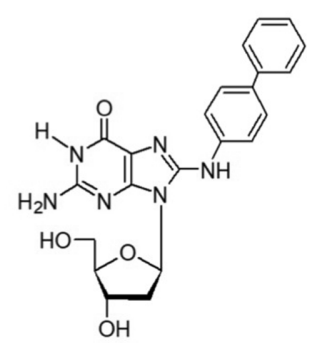

dG-C8-ABP

Fig. 1. Structures of $d G-C 8-A B A, d G-N^{2}-A B A, d A-N^{6}-A B A$, and $d G-C 8-A B P$. 
servative filling (DNA synthesis) of the gap (removed region). NER is performed by several proteins including various xeroderma pigmentosum (XP) family proteins, the transcription factor TFIIH complex, DNA helicase, polymerase $\delta / \varepsilon$, and ligase with their accessary proteins $(15,16)$. Small lesions such as alkyl and hydroxyl adducts are removed by another pathway, base excision repair (BER) $(17,18)$. In BER, the damaged base is excised by DNA glycosylase. The excision of an abasic deoxynucleotide from the DNA strand is performed by apurinic/apyrimidinic (AP) endonuclease, and a correct deoxynucleotide is inserted into the AP site by DNA polymerase $\beta$ and some accessary proteins. Both NER and BER fix damaged DNA accurately without induction of mutations during their processes.

\section{TLS SYSTEM FOR BYPASSING DNA ADDUCTS}

When genomic DNA containing bulky adducts proceeds to replication, the replication fork is stalled at each adduct, leading to cell death. To avoid cell death, cells have mechanisms to complete DNA replication by bypassing these adducts. There are two mechanisms for DNA replication bypass: one is damage avoidance including replication fork regression and recombination repair, which are error-free mechanisms; the other is TLS, which is either error-free or error-prone depending on the adduct structure and the DNA polymerases involved $(19,20)$. The DNA polymerases participating in TLS are translesion DNA polymerases, which are different from the replicative DNA polymerases $\delta$ and $\varepsilon$. The major translesion DNA polymerases are Pol $\eta, \kappa, 1$, and $\zeta$ and Rev1 $(21,22)$. When a DNA replication fork is stalled at a DNA adduct, mono-ubiquitination of proliferative cell nuclear antigen (PCNA) by Rad6/Rad18 proteins triggers the replacement of Pol $\delta$ or $\varepsilon$ with Pol $\eta, \kappa, l$, or $\zeta$ or Rev1, which continues DNA synthesis over the adduct $(19,20)$. Given that translesion DNA polymerases are deficient in 3' to $5^{\prime}$ exonuclease activity (proofreading activity), these polymerases readily insert an incorrect nucleotide opposite the adducted nucleotide and cause a base-change mutation.

\section{CELL-FREE TLS ANALYSIS (IN VITRO TLS ASSAY)}

To measure the activity of each polymerase in terms of TLS over various bulky adducts, in vitro cell-free assays have been performed in many studies (23-28). Such an assay is composed of an oligonucleotide containing a bulky adduct at a specific site, a 5' [ $\left.{ }^{32} \mathrm{P}\right]$ labeled primer, four types of deoxynucleotides, and one purified DNA polymerase. Semiconservative DNA extension from the $\left[{ }^{32} \mathrm{P}\right]$ labeled primer across the adduct, extension arrest at the adduct, and incorporated nucleotides opposite the adduct are visualized by polyacrylamide gel electrophoresis and autoradiography.

In vitro TLS assays have revealed that $\mathrm{Pol} \eta$ and 1 enable a slow bypass over an $N^{2}$ adduct of BPDE or 2-amino-3- methylimidazo-[4,5-f]quinoline (IQ) with guanine; these adducts result in high frequencies of nucleotide misincorporation (28-30). IQ is a heterocyclic aromatic amine formed by cooking of meat or fish. Pol $\kappa$ performs accurate and reasonably efficient bypass over the dG- $N^{2}$-BPDE and dG$N^{2}$-IQ adducts. On the other hand, Pol $\eta$ allows for an efficient error-free bypass over dG-C8-IQ, dG-C8-ABA, or $\mathrm{N}$ [deoxyguanosine-8-yl]-1-aminopyrene (dG-C8-AP). dG$\mathrm{C} 8 \mathrm{-AP}$ is an aminopyrene adduct formed by the diesel exhaust-derived air pollutant 1-nitropyrene (31-35). An inefficient and error-prone bypass over dG-C8-IQ or dGC8-AP is performed by Pol $\kappa$ and $\zeta(31)$ and by Pol $\mathrm{l}$ and $\kappa$ $(34,35)$, respectively. Pol $\kappa$ enables an efficient and errorfree bypass, whereas Pol 1 allows for an inefficient and error-prone bypass over dG-C8-ABA $(32,33)$. Unlike the $\mathrm{dG}-\mathrm{N}^{2}$ adducts, the error-free and error-prone patterns of TLS over dG-C8 adducts are complicated.

\section{DEVELOPMENT OF A TLS ANALYSIS IN MAMMALIAN CELLS (IN VIVO TLS ASSAY)}

In vitro TLS assays may not accurately reflect cellular responses to these adducts because these assays involve limited components as described above. PCNA and replication protein A (RP-A) are essential for DNA replication in the cell but are not included in in vitro assays. PCNA and RP-A assist DNA polymerases to efficiently bypass adducts in the assays (36). Therefore, cell-based TLS assays (in vivo TLS assays) using shuttle vector plasmids with these adducts have been established by several research groups.

Moriya and colleagues constructed a single-stranded pM2-based plasmid that carries a $\sim 15$-mer oligonucleotide with a site-specifically created single bulky adduct (37-39). The plasmid has a replication origin of simian virus 40 (SV40) and replicates in simian COS-7 cells. They also observed error-prone and error-free TLS over the adducts with various endogenous reactive oxygen species $(38,40)$. Yasui et al. (41) investigated TLS over the acetylaminofluorene (AAF) adducts, dG- $N^{2}-\mathrm{AAF}$ and dG-C8-AAF, by a similar method and found that a single dG- $N^{2}$-AAF adduct reduces replication efficiency more than dG-C8-AAF does. Both adducts promote $\mathrm{G}$ to $\mathrm{T}$ transversion, with $\mathrm{dG}-\mathrm{N}^{2}$ AAF being less mutagenic than dG-C8-AAF (41).

Livneh and colleagues $(42,43)$ and Basu and colleagues $(31,44-46)$ used another in vivo TLS assay. They constructed hetero-duplex gap-lesion plasmids using two types of plasmids of different lengths. A site-specifically created single bulky adduct is located in a single-strand gap region ( $\sim 16$ nucleotides). The gap region is filled by TLS after the plasmids are introduced into human cells. They studied TLS over various kinds of bulky adducts in human cells. Basu and colleagues found that Pol $\eta, \kappa, 1$, and $\zeta$ and Rev1 each plays a role in TLS over dG-C8-ABA in human cells in which TLS polymerases were knocked down by siRNA 
(46). Pol $\eta$ and $\kappa$ are considered the major contributors to error-prone TLS over dG-C8-ABA. Rev1 is also important for mutagenesis but may perform a noncatalytic function by physically interacting with the other two TLS polymerases. In contrast, pol $\zeta$ appears to be involved in the error-free bypass of the adduct.

\section{IN VIVO TLS REPORTER ASSAY SYSTEM}

Our method for the in vivo TLS analysis is based on that originally developed for studies on TLS in Escherichia coli $(47,48)$. The constructed shuttle vector plasmid is composed of a lacZ mutation marker gene, ColE1 replication origin, ampicillin resistance gene (marker), an SV40 replication origin, and the $\mathrm{T}$ antigen gene, and the plasmid can replicate in human and E. coli cells (48). In the plasmid lac $Z$ gene, a bulky adduct is created site-specifically on one DNA strand. The other DNA strand without the bulky adduct has a two-base bulge, and thus is two bases longer than the strand with the adduct (Fig. 2). When the DNA strand with the adduct replicates in a human cell, the progenitor plasmids extracted from the cells form blue E. coli colonies on agar plates containing ampicillin, 5-bromo-4chloro-3-indolyl- $\beta$-D-galactopyranoside (X-gal), and isopropyl- $\beta$-D-thiogalactopyranoside (IPTG). When the other DNA strand without the adduct replicates in the cell, the progenitor plasmids yield white $E$. coli colonies on the plates. The proportion of blue colonies among all colonies from the plasmids with adducts is divided by the proportion of those resulting from the plasmids without the adducts; the resulting number represents the rate of TLS over the adducts. Via sequencing of the $l a c Z$ gene in the plasmids extracted from the blue colonies, the frequency and types of mutations caused by the adducts can be elucidated. In these assays, SV40-transformed cells derived from a patient with xeroderma pigmentosum complementation group A (XPA) are used because XPA cells lack the nucleotide excision repair system and cannot remove bulky adducts from plasmids.

\section{(A)}

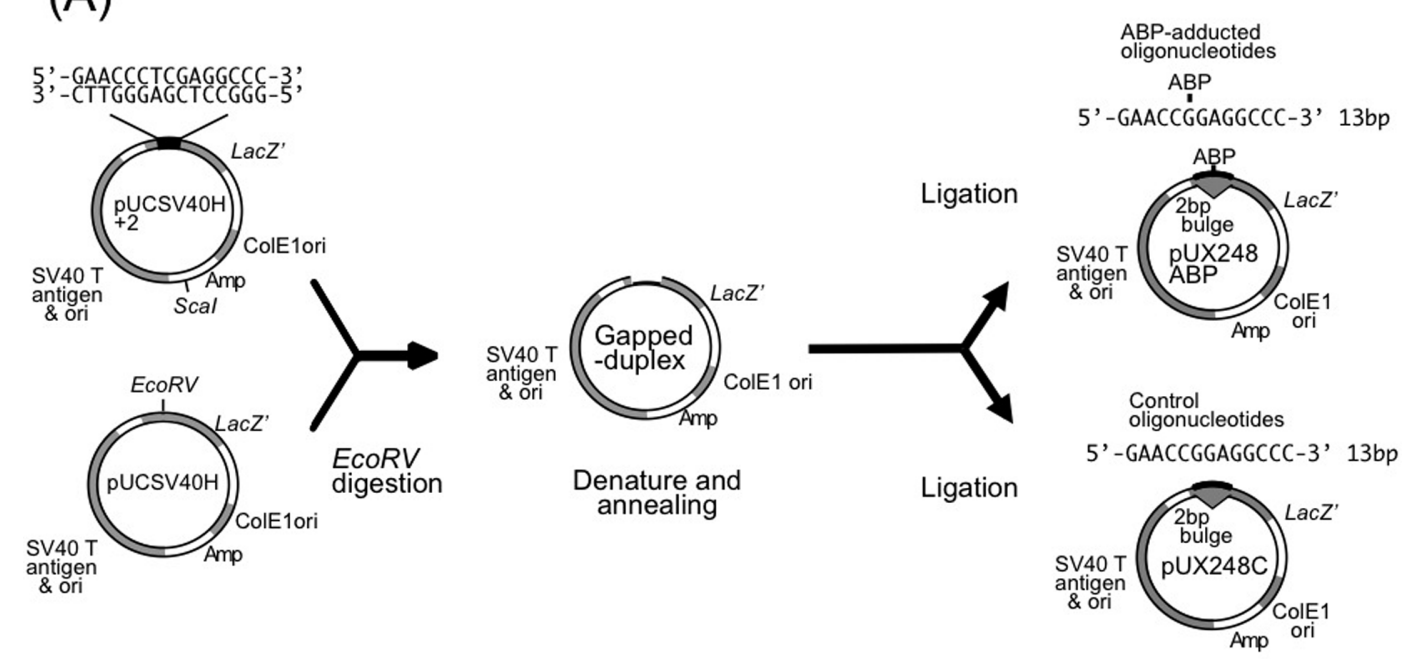

(B)

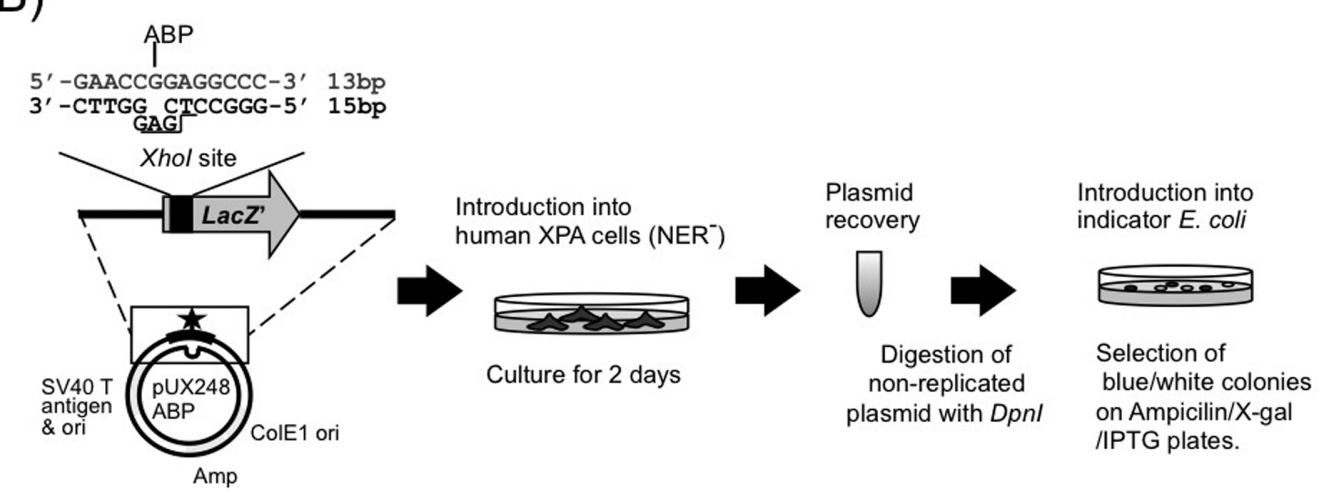

Fig. 2. Methods for the construction of site-specifically dG-C8-ABP-adducted plasmids (A) and the in vivo TLS reporter assay (B). To construct NBA-adducted plasmids, CCTTCCG*TCTCCC is used for dG- $N^{2}-A B A$ and dG-C8-ABA, and CCTTCCA*TCTCCC is applied for dA$N^{6}$-ABA ( $G^{*}$ and $A^{*}$ are ABA-adducted bases). 
(A)

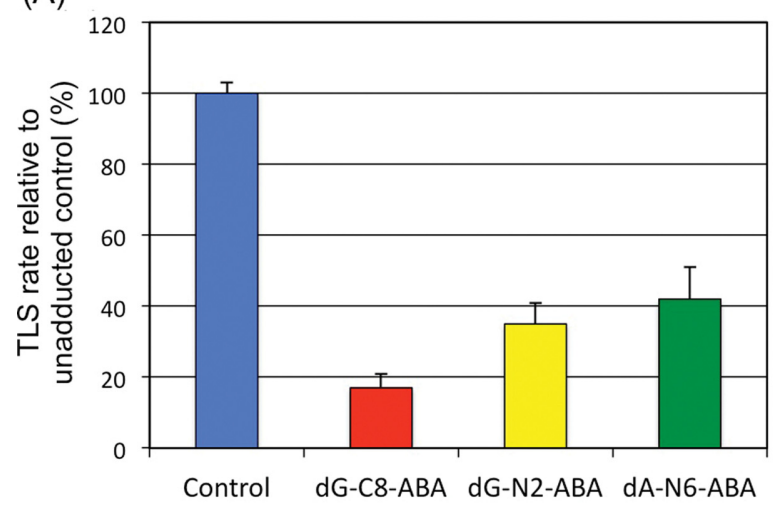

(B)

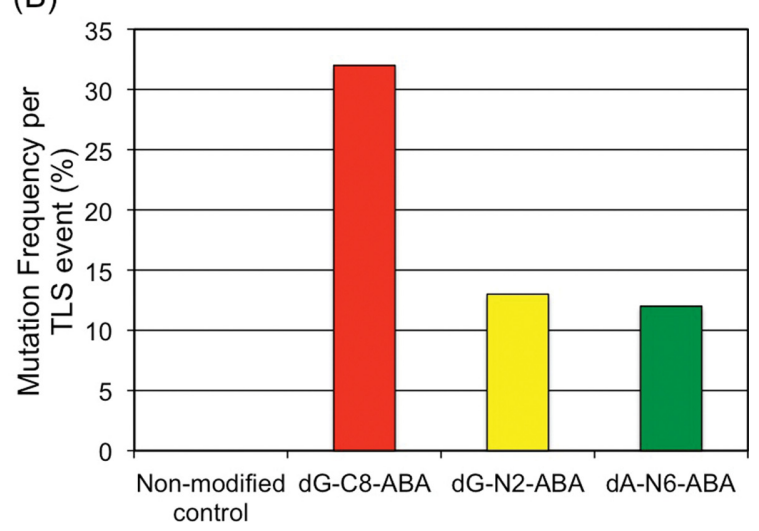

Fig. 3. Rates of TLS over different NBA adducts (A), and frequencies of mutations of the adducted bases (B). The NBA adducts, dG- $N^{2}-$ $A B A$ and dG-C8-ABA are formed in CCTTCCG*TCTCCC, while dA-N $N^{6}-A B A$ is formed in CCTTCCA*TCTCCC (G* and $A^{*}$ are adducted bases).

\section{MUTATIONS ARISING FROM DIFFERENT ADDUCTS INDUCED BY NITROBENZANTHRONE IN HUMAN CELLS}

TLS over three major NBA adducts, dG- $N^{2}-\mathrm{ABA}$, dG$\mathrm{C} 8-\mathrm{ABA}$, and $\mathrm{dA}-N^{6}-\mathrm{ABA}$, in XPA cells was analyzed with our in vivo TLS reporter assay system (9). The assay showed that dG-C8-ABA strongly blocks plasmid replication, while dG- $N^{2}$-ABA and dA- $N^{6}$-ABA block replication moderately (Fig. 3). Mutations occur more frequently during TLS over dG-C8-ABA than during TLS over dG$N^{2}$-ABA or dA- $N^{6}$-ABA. The types of induced mutations are $\mathrm{G}$ to $\mathrm{T}$ transversion at $\mathrm{dG}-N^{2}-\mathrm{ABA}, \mathrm{G}$ to $\mathrm{A}$ and $\mathrm{T}$ base substitutions at dG-C8-ABA, and $A$ to $G$ transition at dA$N^{6}$-ABA. NBA yields dG-C8-ABA more abundantly in cells, and dG-C8-ABA blocks DNA replication and causes

(A)

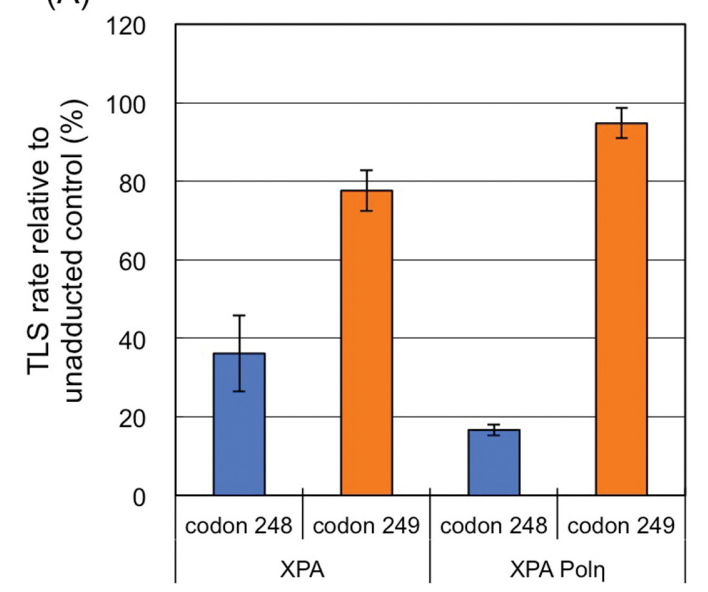

mutations more effectively than the other adducts do; therefore, dG-C8-ABA may be the DNA damage that causes NBA mutagenesis and/or carcinogenesis most frequently (9).

In contrast to human cells, the rate of TLS over $\mathrm{dG}-\mathrm{N}^{2}$ ABA is markedly reduced when the adducted plasmid is propagated in $E$. coli, even under SOS-induced conditions, and $\mathrm{dG}-N^{2}$-ABA induces $\mathrm{G}$ to $\mathrm{T}$ mutations. Other ABA adducts are bypassed in a less mutagenic manner (49). These findings reveal host species differences in the ability to bypass these adducts.

\section{MUTATIONS ARISING FROM AMINOBIPHENYL- GUANINE ADDUCTS IN DIFFERENT DNA SEQUENCES}

TLS over dG-C8-ABP in different DNA sequences has

(B)

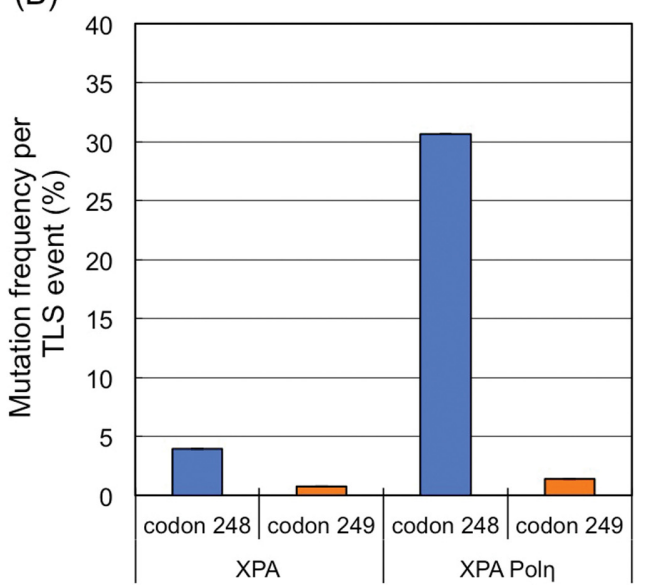

Fig. 4. Rates of TLS over $A B P$ adducts formed on guanine in different sequences $(A)$, and frequencies of mutations of the adducted bases (B) in XPA cells and those overexpressing Pol $\eta$. dG-C8-ABP is formed in CCG*GAGGC and CCGGAG*GCC (G* is the adducted base), which are codon 248 and 249 sequences, respectively, of the human $p 53$ tumor suppressor gene. 
been examined in our in vivo TLS reporter assay system. $\mathrm{ABP}$ is attached to the $\mathrm{C} 8$-guanine of $\mathrm{CCG}^{*} \mathrm{GAGGC}$ and CCGGAG*GCC ( $\mathrm{G}^{*}$ is the adducted base), which represent codon 248 and 249 sequences of the human $p 53$ tumor suppressor gene, respectively. Codons 248 and 249 are a mutation hot spot and cold spot of the p53 gene, respectively, in bladder cancer, whereas codon 248 is a hot spot of ABP adduct formation (14).

The dG-C8-ABP of codon 248 inserted into the lac Z gene strongly blocks plasmid replication, whereas that of codon 249 blocks replication moderately in human XPA cells (Fig. 4). Mutations are more frequently induced in codon 248 than in codon 249. The major induced mutation is $\mathrm{G}$ to $\mathrm{T}$ transversion (50). These findings indicate that the efficiency of TLS over dG-C8-ABP is affected by the surrounding sequences. The mutation distribution in the $p 53$ gene in ABP-induced bladder cancer may be affected by TLS efficiency over the adducts as well as the efficiency of adduct formation in the $p 53$ sequence.

In our in vivo TLS reporter assay, Pol $\eta$ overexpression forced by the transfection of $\mathrm{Pol} \eta$ expression plasmids into XPA cells potentiates DNA replication block and mutation induction at dG-C8-ABP in codon 248 of the $p 53$ gene (Fig. 4 ), and induces $\mathrm{G}$ to A transition as well as $\mathrm{G}$ to $\mathrm{T}$ transversion at the adduct (50). This finding suggests that Pol $\eta$ is involved in error-prone TLS over dG-C8-ABP in codon 248 of the $p 53$ gene in human cells.

\section{CONCLUSIONS}

Aryl hydrocarbons such as NBA, ABP, AAF, BaP, and NP are metabolically activated to reactive forms in certain mammalian cells and preferentially bind covalently to the $N^{2}$ or C8 position of guanine, thus forming a bulky DNA adduct. Such adducts are subjected to TLS to avoid cell death. TLS is performed by Pol $\eta, \kappa, \imath$, and $\zeta$ and Rev1 in an error-free or error-prone manner in the cell. Regarding the NBA adducts (dG- $N^{2}-\mathrm{ABA}$ and dG-C8-ABA), the in vivo gap-lesion TLS assay suggests that $\mathrm{Pol} \eta$ and $\kappa$ are the major contributors to error-prone TLS over dG-C8-ABA, whereas $\mathrm{Pol} \zeta$ seems to participate in error-free bypass over the adduct (46). Our in vivo TLS reporter assay indicates that dG- $N^{2}$-ABA blocks DNA replication less than dG-C8ABA does and is less error-prone than dG-C8-ABA in human XPA cells. dG-C8-ABA mainly causes $G$ to T transversion (9). The research on TLS polymerases involved in error-free or error-prone bypass over $\mathrm{dG}-N^{2}-\mathrm{ABA}$ and $\mathrm{dG}-$ C8-ABA is in progress in TLS polymerase-deficient cells created by a genome-editing method. Our preliminary findings suggest that $\mathrm{dG}-N^{2}$-ABA is mainly bypassed by Pol $\kappa$ in an error-free manner, whereas dG-C8-ABA is bypassed by Pol $\eta$ in an error-free manner, but by Pol $\kappa$ in an errorprone manner.

The stronger contribution of the $\mathrm{C} 8$ guanine adduct to the replication block and error-prone TLS than that of the $N^{2}$ guanine adduct may not be a universal phenomenon. dG$N^{2}$-IQ blocks DNA replication more strongly than dG-C8IQ does. Pol $\eta$ is mainly involved in the bypass over dG- $N^{2}$ IQ and dG-C8-IQ in an error-prone and error-free manner, respectively (30,31). dG-C8-AAF blocks DNA replication slightly more strongly than $\mathrm{dG}-N^{2}$-AAF does, and the bypass of dG-C8-AAF is more error-prone than that of dG$N^{2}$-AAF (41). dG-C8-AAF allows for a higher frequency of nucleotide misincorporation than $\mathrm{dG}-N^{2}$-AAF does when Pol $\eta$ and $\kappa$ are employed in in vitro TLS assays (41). The inconsistency of the TLS efficiency over C8 guanine adducts may be due to differences in the adducted molecules, assay systems, and host mammalian cells used in three laboratories.

According to our in vivo reporter assay of TLS over dGC8-ABP, it is important to note that TLS efficiency and error-proneness are affected by the surrounding DNA sequences of the adduct (50). Elucidation of the general mechanisms determining the efficiency, error-proneness, and participating polymerases of TLS over these adducts represents the next step in the research into TLS. These studies will clarify the mechanisms underlying aryl hydrocarbon mutagenesis and carcinogenesis.

\section{ACKNOWLEDGMENTS}

This article describes the content of TY's plenary lecture at the Spring International Symposium of the Korean Societies of Toxicology and Environmental Mutagens held in May 2017. We sincerely thank Drs. Young-Rok Seo, Michael Lee, and Youngkyu Lee for inviting TY to the symposium. The publication of this article is supported by the basic research grant from Osaka Prefecture University.

\section{CONFLICT OF INTEREST}

The authors declare that they have no competing interests.

Received September 3, 2017; Revised September 20, 2017; Accepted September 25, 2017

\section{REFERENCES}

1. Choi, H., Harrison, R., Komulainen, H. and Saborit, J.M.D. (2010) Polycyclic aromatic hydrocarbons in WHO Guidelines for Indoor Air Quality: Selected Pollutants. World Health Organization, Geneva, pp. 299-346.

2. Baird, W.M., Hooven, L.A. and Mahadevan, B. (2005) Carcinogenic polycyclic aromatic hydrocarbon-DNA adducts and mechanism of action. Environ. Mol. Mutagen., 45, 106-114.

3. Alexandrov, K., Rojas, M. and Satarug, S. (2010) The critical DNA damage by benzo(a)pyrene in lung tissues of smokers and approaches to preventing its formation. Toxicol. Lett., 198, 63-68. 
4. Shiizaki, K., Kawanishi, M. and Yagi, T. (2017) Modulation of benzo[a]pyrene-DNA adduct formation by CYP1 inducer and inhibitor. Genes Environ., 39, 14.

5. Takamura-Enya, T., Suzuki, H. and Hisamatsu, Y. (2006) Mutagenic activities and physicochemical properties of selected nitrobenzanthrones. Mutagenesis, 21, 399-404.

6. Arlt, V.M., Schmeiser, H.H., Osborne, M.R., Kawanishi, M., Kanno, T., Yagi, T., Phillips, D.H. and Takamura-Enya, T. (2006) Identification of three major DNA adducts formed by the carcinogenic air pollutant 3-nitrobenzanthrone in rat lung at the $\mathrm{C} 8$ and $N^{2}$ position of guanine and at the N6 position of adenine. Int. J. Cancer, 118, 2139-2146.

7. Takamura-Enya, T., Kawanishi, M., Yagi, T. and Hisamatsu, Y. (2007) Structural identification of DNA adducts derived from 3-nitrobenzanthrone, a potent carcinogen present in the atmosphere. Chem. Asian J., 2, 1174-1185.

8. Kanno, T., Kawanishi, M., Takamura-Enya. T., Arlt, V.M., Phillips, D.H. and Yagi, T. (2007) DNA adduct formation in human hepatoma cells treated with 3-nitrobenzanthrone: analysis by the ${ }^{32} \mathrm{P}$-postlabeling method. Mutat. Res., 634, 184191.

9. Kawanishi, M., Fujikawa, Y., Ishii, H., Nishida, H., Higashigaki, Y., Kanno, T., Matsuda, T., Takamura-Enya, T. and Yagi, T. (2013) Adduct formation and repair, and translesion DNA synthesis across the adducts in human cells exposed to 3nitrobenzanthrone. Mutat. Res., 753, 93-100.

10. Nagy, E., Zeisig, M., Kawamura, K., Hisamatsu, Y., Sugeta, A., Adachi, S. and Möller, L. (2005) DNA adduct and tumor formations in rats after intratracheal administration of the urban air pollutant 3-nitrobenzanthrone. Carcinogenesis, 26, 18211828.

11. Cohen, S.M., Boobis, A.R., Meek, M.E., Preston, R.J. and McGregor, D.B. (2006) 4-Aminobiphenyl and DNA reactivity: case study within the context of the 2006 IPCS Human Relevance Framework for Analysis of a cancer mode of action for humans. Crit. Rev. Toxicol., 36, 803-819.

12. IARC (1987) 4-Aminobiphenyl in Overall Evaluations of Carcinogenicity. IARC Monographs on the Evaluation of Carcinogenic Risk of Chemicals to Humans (Suppl. 7). International Agency for Research on Cancer, Lyon, France, pp. 91-92.

13. Besaratinia, A. and Tommasi, S. (2013) Genotoxicity of tobacco smoke-derived aromatic amines and bladder cancer: current state of knowledge and future research directions. FASEB J., 27, 2090-2100.

14. Feng, Z., Hu, W., Rom, W.N., Beland, F.A. and Tang, M.S. (2002) 4-aminobiphenyl is a major etiological agent of human bladder cancer: evidence from its DNA binding spectrum in human p53 gene. Carcinogenesis, 23, 1721-1727.

15. Fuss, J.O. and Cooper, P.K. (2006) DNA repair: dynamic defenders against cancer and aging. PLoS Biol., 4, e203.

16. Spivak, G. (2015) Nucleotide excision repair in humans. $D N A$ Repair (Amst.), 36, 13-18.

17. Bauer, N.C., Corbett, A.H. and Doetsch, P.W. (2015) The current state of eukaryotic DNA base damage and repair. Nucleic Acids Res., 43, 10083-10101.

18. Carter, R.J. and Parsons, J.L. (2016) Base excision repair, a pathway regulated by posttranslational modifications. Mol. Cell. Biol., 36, 1426-1437.
19. Sale, J.E. (2013) Translesion DNA synthesis and mutagenesis in eukaryotes. Cold Spring Harb. Perspect. Biol., 5, a012708.

20. Basu, A.K., Pande, P. and Bose, A. (2017) Translesion synthesis of 2'-deoxyguanosine lesions by eukaryotic DNA polymerases. Chem. Res. Toxicol., 30, 61-72.

21. Livneh, Z., Ziv, O. and Shachar, S. (2010) Multiple two-polymerase mechanisms in mammalian translesion DNA synthesis. Cell Cycle, 9, 729-735.

22. Goodman, M.F. and Woodgate, R. (2013) Translesion DNA polymerases. Cold Spring Harb. Perspect. Biol., 5, a010363.

23. Kirouac, K.N., Basu, A.K. and Ling, H. (2013) Replication of a carcinogenic nitropyrene DNA lesion by human Y-family DNA polymerase. Nucleic Acids Res., 41, 2060-2071.

24. Shibutani, S., Suzuki, N. and Grollman, A.P. (2004) Mechanism of frameshift (deletion) generated by acetylaminofluorene-derived DNA adducts in vitro. Biochemistry, 43, 1592915935.

25. Schorr, S., Schneider, S., Lammens, K., Hopfner, K.P. and Carell, T. (2010) Mechanism of replication blocking and by pass of Y-family polymerase $\eta$ by bulky acetylaminofluorene DNA adducts. Proc. Natl. Acad. Sci. U.S.A., 107, 2072020725.

26. Suzuki, N., Ohashi, E., Kolbanovskiy, A., Geacintov, N.E., Grollman, A.P., Ohmori. H. and Shibutani, S. (2002) Translesion synthesis by human DNA polymerase $\kappa$ on a DNA template containing a single stereoisomer of dG-(+)- or dG-(-)anti- $N^{2}$-BPDE (7,8-dihydroxy-anti-9,10-epoxy-7,8,9,10-tetrahydrobenzo[a]pyrene). Biochemistry, 41, 6100-6106.

27. Choi, J.Y., Angel, K.C. and Guengerich, F.P. (2006) Translesion synthesis across bulky $N^{2}$-alkyl guanine DNA adducts by human DNA polymerase к. J. Biol. Chem., 281, 21062-21072.

28. Frank, E.G., Sayer, J.M., Kroth, H., Ohashi, E., Ohmori, H., Jerina, D.M. and Woodgate, R. (2002) Translesion replication of benzo[a]pyrene and benzo[c]phenanthrene diol epoxide adducts of deoxyadenosine and deoxyguanosine by human DNA polymerase iota. Nucleic Acids Res., 30, 5284-5292.

29. Chiapperino, D., Kroth, H., Kramarczuk, I.H., Sayer, J.M., Masutani, C., Hanaoka, F., Jerina, D.M. and Cheh, A.M. (2002) Preferential misincorporation of purine nucleotides by human DNA polymerase eta opposite benzo[a]pyrene 7,8-diol 9,10-epoxide deoxyguanosine adducts. J. Biol. Chem., 277, 11765-11771.

30. Bose, A., Millsap, A.D., DeLeon, A., Rizzo, C.J. and Basu, A.K. (2016) Translesion synthesis of the $N^{2}-2$ '-deoxyguanosine adduct of the dietary mutagen IQ in human cells: Errorfree replication by DNA polymerase $\kappa$ and mutagenic bypass by DNA polymerases $\eta, \zeta$, and Rev1. Chem. Res. Toxicol., 29, 1549-1559.

31. Bose, A., Pande, P., Jasti, V.P., Millsap, A.D., Hawkins, E.K., Rizzo, C.J. and Basu, A.K. (2015) DNA polymerases $\kappa$ and $\zeta$ cooperatively perform mutagenic translesion synthesis of the C8-2'-deoxyguanosine adduct of the dietary mutagen IQ in human cells. Nucleic Acids Res., 43, 8340-8351.

32. Tokarsky, E.J., Gadkari, V.V., Zahurancika, W.J., Malik, C.K., Basu, A.K. and Suo, Z. (2016) Pre-steady-state kinetic investigation of bypass of a bulky guanine lesion by human Y-family DNA polymerases. DNA Repair (Amst.), 46, 20-28.

33. Patra, A., Politica, D.A., Chatterjee, A., Tokarsky, E.J., Suo, Z., Basu, A.K., Stone, M.P. and Egli, M. (2016) Mechanism of 
error-free bypass of the environmental carcinogen N-(2'-deoxyguanosin-8-yl)-3-aminobenzanthrone adduct by human DNA polymerase $\eta$. Chembiochem, 17, 2033-2037.

34. Sherrer, S.M., Sanman, L.E., Xia, C.X., Bolin, E.R., Malik, C.K., Efthimiopoulos, G., Basu, A.K. and Suo, Z. (2012) Kinetic analysis of the bypass of a bulky DNA lesion catalyzed by human Y-family DNA polymerases. Chem. Res. Toxicol., 25, 730-740.

35. Sherrer, S.M., Taggart, D.J., Pack, L.R., Malik, C.K., Basu, A.K. and Suo, Z. (2012) Quantitative analysis of the mutagenic potential of 1-aminopyrene-DNA adduct bypass catalyzed by Y-family DNA polymerases. Mutat. Res., 737, 25-33.

36. Maga, G., Villani, G., Crespan, E., Wimmer, U., Ferrari, E., Bertocci, B. and Hübscher, U. (2007) 8-oxo-guanine bypass by human DNA polymerases in the presence of auxiliary proteins. Nature, 447, 606-608.

37. Moriya, M. (1993) Single-stranded shuttle phagemid for mutagenesis studies in mammalian cells: 8-oxoguanine in DNA induces targeted G.C-->T.A transversions in simian kidney cells. Proc. Natl. Acad. Sci. U.S.A., 90, 1122-1126.

38. Yang, I.Y., Miller, H., Wang, Z., Frank, E.G., Ohmori, H., Hanaoka, F. and Moriya, M. (2003) Mammalian translesion DNA synthesis across an acrolein-derived deoxyguanosine adduct. Participation of DNA polymerase $\eta$ in error-prone synthesis in human cells. J. Biol. Chem., 278, 13989-13994.

39. Hashimoto, K., Bonala, R., Johnson, F., Grollman, A.P. and Moriya, M. (2016) Y-family DNA polymerase-independent gap-filling translesion synthesis across aristolochic acidderived adenine adducts in mouse cells. DNA Repair (Amst.), 46, 55-60.

40. Pollack, M., Yang, I.Y., Kim, H.Y., Blair, I.A. and Moriya, M. (2006) Translesion DNA Synthesis across the heptanone-etheno-2'-deoxycytidine adduct in cells. Chem. Res. Toxicol., 19, 1074-1079.

41. Yasui, M., Dong, H., Bonala R.R., Suzuki, N., Ohmori, H., Hanaoka, F., Johnson, F., Grollman, A.P. and Shibutani S. (2004) Mutagenic properties of 3-(deoxyguanosin- $\left.N^{2}-y l\right)-2-$ acetylaminofluorene, a persistent acetylaminofluorene-derived DNA adduct in mammalian cells. Biochemistry, 43, 15005-
15013.

42. Avkin, S. and Livneh, Z. (2002) Efficiency, specificity and DNA polymerase-dependence of translesion replication across the oxidative DNA lesion 8-oxoguanine in human cells. Mutat. Res., 510, 81-90.

43. Avkin, S., Goldsmith, M., Velasco-Miguel, S., Geacintov, N., Friedberg, E.C. and Livneh, Z. (2004) Quantitative analysis of translesion DNA synthesis across a benzo[a]pyrene-guanine adduct in mammalian cells: the role of DNA polymerase $\kappa . J$. Biol. Chem., 279, 53298-53305.

44. Watt, D.L., Utzat, C.D., Hilario, P. and Basu, A.K. (2007) Mutagenicity of the 1-nitropyrene-DNA adduct N-(deoxyguanosin-8-yl)-1-aminopyrene in mammalian cells. Chem. Res. Toxicol., 20, 1658-1664.

45. Tolentino, J.H., Burke, T.J., Mukhopadhyay, S., McGregor, W.G. and Basu, A.K. (2008) Inhibition of DNA replication fork progression and mutagenic potential of $1, N^{6}$-ethenoadenine and 8-oxoguanine in human cell extracts. Nucleic Acids Res., 36, 1300-1308.

46. Pande, P., Malik, C.K., Bose, A., Jasti, V.P. and Basu, A.K. (2014) Mutational analysis of the C8-guanine adduct of the environmental carcinogen 3-nitrobenzanthrone in human cells: critical roles of DNA polymerases $\eta$ and $\kappa$ and Rev1 in errorprone translesion synthesis. Biochemistry, 53, 5323-5331.

47. Veaute, X. and Fuchs, R. (1993) Greater susceptibility to mutations in lagging strand of DNA replication in Escherichia coli than in leading strand. Science, 261, 598-600.

48. Sawai, T., Kawanishi, M., Takamura-Enya, T. and Yagi, T. (2009) Establishment of a method for analyzing translesion DNA synthesis across a single bulky adduct in human cells. Genes Environ., 31, 24-30.

49. Kawanishi, M., Kanno, T., Nishida, H., Takamura-Enya, T. and Yagi, T. (2013) Translesion DNA synthesis across various DNA adducts produced by 3-nitrobenzanthrone in Escherichia coli. Mutat. Res., 754, 32-38.

50. Sawai, T., Kawanishi, M., Takamura-Enya, T. and Yagi, T. (2009) Mutations mediated by translesion DNA synthesis over 4-aminobiphenyl adducts in human cells. Radiat. Biol. Commun., 44, 274-284 (in Japanese). 